\title{
Impact of WiMAX Interference on MB-OFDM UWB Systems: Analysis and Mitigation*
}

\author{
Chris Snow ${ }^{\dagger}$, Lutz Lampe, and Robert Schober \\ Department of Electrical and Computer Engineering \\ The University of British Columbia \\ Vancouver, British Columbia, Canada \\ Email: \{csnow, lampe, rschober\}@ece.ubc.ca
}

\begin{abstract}
This paper presents an analysis of the performance of the Multiband Orthogonal Frequency Division Multiplexing (MB-OFDM) system for Ultra Wideband (UWB) communication in the presence of interference from IEEE 802.16 WiMAX systems operating in the $3.5 \mathrm{GHz}$ band. We present an exact analysis of the uncoded bit error rate (BER) of the MB-OFDM system, based on Laplace transform techniques. We also present a simple Gaussian approximation for the WiMAX interference, and establish its relative accuracy and usefulness by means of comparison with the exact analysis and simulations. Such a Gaussian approximation is especially useful for simplified performance analysis, as well as for the design of interference mitigation techniques. Motivated by the Gaussian approximation, we propose a simple two-stage interference mitigation technique for coded MB-OFDM transmissions, consisting of interference spectrum estimation during silent periods followed by appropriate bit metric weighting during Viterbi decoding. We compare parametric and non-parametric spectrum estimation techniques for various scenarios of interest. In the presence of WiMAX interference, the two-stage interference mitigation provides substantial gains in performance in return for modest increases in complexity and without requiring any modifications to the MBOFDM transmitter or signal structure.
\end{abstract}

Index terms: Multiband orthogonal frequency division multiplexing (OFDM), ultra wideband (UWB), WiMAX, narrowband interference, interference suppression, spectral estimation, interference mitigation.

* This work will be presented in part at the 2007 IEEE International Conference on Ultra-Wideband (ICUWB), and was presented in part at the 2007 IEEE Pacific Rim Conference on Communications, Computers and Signal Processing (PACRIM). The completion of this research was made possible thanks to Bell Canada's support through its Bell University Laboratories R\&D program and the Natural Sciences and Engineering Research Council of Canada (Grant CRDPJ 320 552), and with the support of a Canada Graduate Scholarship. This research was enabled by WestGrid computing resources, funded in part by the Canada Foundation for Innovation, Alberta Innovation and Science, BC Advanced Education, and participating institutions. Equipment is provided by IBM, HP, and SGI.

$\dagger$ Corresponding author 


\section{Introduction}

In this paper, we consider the ECMA-368 Multiband Orthogonal Frequency Division Multiplexing (MB-OFDM) standard for high rate Ultra Wideband (UWB) wireless communication in the 3.110.6 $\mathrm{GHz}$ band $[1,2]$. Because UWB systems in this band are operating as spectral underlay systems $[3,4]$, they will unavoidably be impacted by the transmissions of incumbent systems. We consider as interferer the WiMAX IEEE 802.16 system for wireless metropolitan area networks, operating in the licensed 3.5 GHz band [5]. The WiMAX standard consists of both single-carrier (SC) and OFDM-based modulation schemes for use below $11 \mathrm{GHz}$. We address both modulation techniques herein.

When WiMAX is deployed in the $3.5 \mathrm{GHz}$ band, it will be a source of interference for MBOFDM systems also using this band. For this reason, there has recently been great interest in coexistence techniques between WiMAX and UWB systems $[6,7]$. Recent work also examines the effects of single-carrier linearly-modulated narrowband interference signals on system design in MBOFDM [8]. The authors of [9] consider the effect of narrowband OFDM interference on time-hopping (TH) and direct-sequence (DS) UWB systems. They have shown that some narrowband OFDM signals can be modeled as Gaussian interference upon the considered UWB systems. However, they do not consider OFDM-based UWB systems (such as MB-OFDM) as victim receivers. It is not immediately clear that such a Gaussian assumption holds for all forms of WiMAX interference to MB-OFDM systems, especially due to the wide range of allowable WiMAX operating bandwidths and the various modulation types. An accurate Gaussian approximation would be beneficial for both simple performance evaluation techniques and the design of interference mitigation strategies, and thus the question of the validity of this approximation motivates our work herein.

We first investigate the effect of a WiMAX system operating in the $3.5 \mathrm{GHz}$ band and causing interference to an MB-OFDM system. In particular, we provide an exact analysis of the effect of the WiMAX system on the uncoded bit error rate (BER) of the MB-OFDM system, based on Laplace transform techniques (Section 3). We then compare the exact analysis with a Gaussian 
approximation for the WiMAX interference signal (Section 4).

Motivated by the approximately Gaussian nature of the WiMAX interference, we propose a simple two-stage interference mitigation technique for coded MB-OFDM transmissions according to the ECMA-368 standard, consisting of interference spectrum estimation during silent periods followed by appropriate bit metric weighting during Viterbi decoding (Section 5). We compare parametric and non-parametric spectrum estimation techniques for coded MB-OFDM transmissions and WiMAX interference for various scenarios of interest (Section 6). The proposed two-stage interference mitigation technique is shown to be highly effective at mitigating the impact of WiMAX interference.

Notation: In this paper, $\Re\{\cdot\}$ and $\Im\{\cdot\}$ denote the real and imaginary parts of a complex number, respectively, $\otimes$ denotes the convolution operator, $\mathbb{E}\{\cdot\}$ denotes expectation, and $Q(\cdot)$ is the Gaussian-Q function [10].

\section{System Model}

In this section, we describe the signal models for the MB-OFDM transmitter and receiver, and for the WiMAX interferer. For both MB-OFDM and WiMAX-OFDM, we adopt the OFDM filterbank model [11]. A block diagram of the system under consideration is given in Figure 1.

\subsection{MB-OFDM Signal Model}

The MB-OFDM transmitted signal is given by

$$
s_{\mathrm{m}}(t)=\sum_{q=-\infty}^{\infty} \sum_{k=0}^{N_{\mathrm{m}}-1} x_{k, q} \phi_{k}\left(t-q T_{\mathrm{m}}\right) e^{j 2 \pi f_{\mathrm{m}} t},
$$

where $N_{\mathrm{m}}, T_{\mathrm{m}}$, and $f_{\mathrm{m}}$ are the number of subcarriers, the OFDM symbol duration, and the carrier frequency $^{1}$, respectively, cf. [1]. The transmitted Quaternary Phase Shift Keying (QPSK) symbols

\footnotetext{
${ }^{1}$ We note that, due to the MB-OFDM frequency hopping, $f_{\mathrm{m}}$ is a function of the MB-OFDM symbol index $q$. However, in the sequel, we will consider the cases of (a) the presence of an in-band WiMAX interferer, and (b) the absence of such an interferer, separately, so we ignore the dependency of $f_{\mathrm{m}}$ on $q$ for the time being.
} 
are denoted by $x_{k, q}$, where $k$ and $q$ represent the subcarrier index and the MB-OFDM symbol index, respectively. The basis function for subcarrier $k$ is given by

$$
\phi_{k}(t)=\left\{\begin{array}{ll}
\frac{1}{\sqrt{D_{\mathrm{m}}}} e^{j 2 \pi Q_{\mathrm{m}} k\left(t-C_{\mathrm{m}}\right)} & \text { if } t \in\left[0, T_{\mathrm{m}}\right] \\
0 & \text { else }
\end{array},\right.
$$

where $C_{\mathrm{m}}, D_{\mathrm{m}}=T_{\mathrm{m}}-C_{\mathrm{m}}, W_{\mathrm{m}}$, and $Q_{\mathrm{m}}=W_{\mathrm{m}} / N_{\mathrm{m}}$ are the durations of the guard interval and the data-carrying part of the OFDM symbol, the bandwidth of transmission, and the bandwidth per subcarrier, respectively, cf. Table 1.

While the MB-OFDM standard incorporates convolutional coding for error correction $[1,2]$, we first focus on uncoded modulation in order to simplify the analysis. Ignoring the coding also allows us to focus on the contribution of the interference to the BER degradation, and to more clearly study possible approximations for the interference signal. We will consider interference mitigation schemes for MB-OFDM with coding according to the ECMA-368 standard in Section 5.

Next, we introduce the WiMAX OFDM and SC signal models.

\subsection{WiMAX-OFDM Signal Model}

The WiMAX-OFDM transmitted signal is given by

$$
s_{\mathrm{n}}(t)=\sum_{\ell=-\infty}^{\infty} \sum_{d=0}^{N_{\mathrm{n}}-1} z_{d, \ell} \theta_{d}\left(t-\ell T_{\mathrm{n}}\right) e^{j 2 \pi f_{\mathrm{n}} t},
$$

where the modulated symbols are denoted by $z_{d, \ell}$. The WiMAX standard specifies Binary Phase Shift Keying (BPSK), QPSK, 16-QAM (Quadrature Amplitude Modulation), and 64-QAM modulation schemes [5]. We consider only BPSK and QPSK in this work because of space limitations, but note that similar analyses can be performed for the QAM schemes and similar results will be observed. All parameters with subscript $\mathrm{n}$ are defined similarly as the equivalent MB-OFDM parameters with subscript $\mathrm{m}$, and are given in Table 1 . The basis function for subcarrier $d$ is given by

$$
\theta_{d}(t)=\left\{\begin{array}{ll}
\frac{1}{\sqrt{D_{\mathrm{n}}}} e^{j 2 \pi Q_{\mathrm{n}} d\left(t-C_{\mathrm{n}}\right)} & \text { if } t \in\left[0, T_{\mathrm{n}}\right] \\
0 & \text { else }
\end{array} .\right.
$$




\subsection{WiMAX-SC Signal Model}

The WiMAX-SC transmitted signal is given by

$$
s_{\mathrm{s}}(t)=\sum_{\ell=-\infty}^{\infty} z_{\ell} p\left(t-\ell T_{\mathrm{s}}\right) e^{j 2 \pi f_{\mathrm{s}} t},
$$

where the modulated symbols are denoted by $z_{\ell}, f_{\mathrm{s}}$ and $T_{\mathrm{s}}$ are the WiMAX-SC carrier frequency and symbol period, respectively, and $p(t)$ denotes the square-root raised cosine pulse shaping filter with roll-off factor 0.25 (cf. [5]). The WiMAX-SC standard specifies BPSK, QPSK, 16-QAM, 64-QAM, and 256-QAM modulation schemes [5]. Again, for sake of space, we consider only BPSK and QPSK in this work, but note that similar analysis can be performed for the QAM schemes and similar results will be observed.

\subsection{Channel Models and Receiver Processing}

The MB-OFDM signal passes through a channel with impulse response $h(t)$. Due to the relatively small WiMAX bandwidth, and for simplicity, we consider a single-tap WiMAX channel with amplitude $A$ and phase offset $\alpha$ uniformly distributed on $[0,2 \pi){ }^{2}$ The received signal, after down-conversion to baseband and assuming that the interference signal lies in the band of interest, is given by

$$
r(t)=\left[s_{\mathrm{m}}(t) \otimes h(t)\right] e^{-j 2 \pi f_{\mathrm{m}} t}+i(t)+n(t)
$$

where $n(t)$ is the complex additive white Gaussian noise (AWGN), and

$$
i(t)=A e^{j \alpha} s_{X}(t-\tau) e^{-j 2 \pi f_{\mathrm{m}} t},
$$

where $X \in\{\mathrm{n}, \mathrm{s}\}$ depending on whether OFDM or SC WiMAX interference is considered, $\tau$ denotes the timing offset of the WiMAX signal, which is uniformly distributed on $\left[0, T_{X}\right]$. For future reference, we define $\Delta=f_{X}-f_{\mathrm{m}}$ as the separation between the carrier frequencies of the two systems.

\footnotetext{
${ }^{2} \mathrm{~A}$ slowly time-varying multipath channel can be incorporated by replacing $\theta_{d}(t)$ and $p(t)$ with $\theta_{d}(t) \otimes g(t)$ and $p(t) \otimes g(t)$, respectively, where $g(t)$ is the short-term channel impulse response.
} 
The baseband processing consists of a filterbank matched to $\phi_{k}(t)$ over $\left[C_{\mathrm{m}}, T_{\mathrm{m}}\right]$, which, for subcarrier $k$, is given by

$$
\psi_{k}(t)=\left\{\begin{array}{ll}
\phi_{k}^{*}\left(T_{\mathrm{m}}-t\right) & \text { if } t \in\left[0, T_{\mathrm{m}}-C_{\mathrm{m}}\right] \\
0 & \text { else }
\end{array} .\right.
$$

Without loss of generality, we consider the MB-OFDM symbol index $q=0$, and the statistic for subcarrier $k$ is given by

$$
r_{k}=\left.\left(r(t) \otimes \psi_{k}(t)\right)\right|_{t=T_{\mathrm{m}}}=\int_{-\infty}^{\infty} r(t) \psi_{k}\left(T_{\mathrm{m}}-t\right) \mathrm{d} t=\tilde{y}_{k}+\tilde{i}_{k}+\tilde{n}_{k},
$$

where $\tilde{y}_{k}, \tilde{i}_{k}$, and $\tilde{n}_{k}$ denote the received signal, interference, and noise terms, respectively. We note that, since the basis functions $\phi_{k}(t)$ are orthogonal,

$$
\tilde{y}_{k}=G_{k} x_{k}
$$

where we have dropped the MB-OFDM symbol index $q=0$, and $G_{k}=g_{k} e^{j \eta_{k}}$ denotes the frequencydomain channel gain of subcarrier $k$, which is the sample of the Fourier transform of $h(t)$ at frequency $\left(f_{\mathrm{m}}+k Q_{\mathrm{m}}\right)$.

We now turn to consider the interference term, which, from (8) and (9), is given by

$$
\tilde{i}_{k}=\int_{C_{\mathrm{m}}}^{T_{\mathrm{m}}} i(t) \phi_{k}^{*}(t) \mathrm{d} t
$$

1) WiMAX-OFDM: The interference term can be expressed as

$$
\tilde{i}_{k}=A e^{j \alpha} \sum_{\ell=-\infty}^{\infty} \sum_{d=0}^{N_{\mathrm{n}}-1} z_{d, \ell} \beta_{k, \ell, d},
$$

where

$$
\beta_{k, \ell, d}=\int_{C_{\mathrm{m}}}^{T_{\mathrm{m}}} \theta_{d}\left(t-\ell T_{\mathrm{n}}-\tau\right) \phi_{k}^{*}(t) e^{j 2 \pi \Delta t} \mathrm{~d} t
$$


By noting that $\theta_{d}\left(t-\ell T_{\mathrm{n}}-\tau\right)$ is a complex exponential on $\left[\ell T_{\mathrm{n}}+\tau, T_{\mathrm{n}}+\ell T_{\mathrm{n}}+\tau\right]$ and zero otherwise, $\beta_{k, \ell, d}$ can be expressed in closed form as

$$
\beta_{k, \ell, d}=\frac{e^{j 2 \pi\left(Q_{\mathrm{m}} C_{\mathrm{m}} k-Q_{\mathrm{n}} C_{\mathrm{n}} d\right)}}{j 2 \pi\left(Q_{\mathrm{n}} d-Q_{\mathrm{m}} k+\Delta\right) \sqrt{D_{\mathrm{m}} D_{\mathrm{n}}}}\left(e^{j 2 \pi\left(Q_{\mathrm{n}} d-Q_{\mathrm{m}} k+\Delta\right) U}-e^{j 2 \pi\left(Q_{\mathrm{n}} d-Q_{\mathrm{m}} k+\Delta\right) L}\right)
$$

where $L=\max \left(C_{\mathrm{m}}, \ell T_{\mathrm{n}}+\tau\right)$, and $U=\min \left(T_{\mathrm{m}}, T_{\mathrm{n}}+\ell T_{\mathrm{n}}+\tau\right)$.

2) WiMAX-SC: In this case, the interference term can be expressed as

$$
\tilde{i}_{k}=A e^{j \alpha} \sum_{\ell=-\infty}^{\infty} z_{\ell} \beta_{k, \ell}
$$

where

$$
\beta_{k, \ell}=\int_{C_{\mathrm{m}}}^{T_{\mathrm{m}}} p\left(t-\ell T_{\mathrm{s}}-\tau\right) \phi_{k}^{*}(t) e^{j 2 \pi \Delta t} \mathrm{~d} t .
$$

We note that (16) can be solved by numerical integration, or can be written in terms of the exponential integral. We omit the latter form due to its notational complexity, but note that it can easily be obtained with a symbolic math package such as Mathematica.

\section{Performance Analysis}

In this section, we provide an analysis of the BER for MB-OFDM in the presence of WiMAX interference. We begin by considering the exact analysis (Section 3.1), followed by a Gaussian approximation (Section 3.2). In Sections 3.3 and 3.4, we present the overall BER expressions including the effects of frequency hopping for the cases of non-fading and fading channels, respectively.

\subsection{Exact BER Analysis with In-Band Interferer}

We start by noting that MB-OFDM employs QPSK modulation, which can also be considered

equivalently as two independent BPSK modulations. As such, and noting that both $\tilde{i}_{k}$ and $\tilde{n}_{k}$ are rotationally symmetric, we can simplify our analysis by considering $x_{k, \ell}$ as BPSK symbols in the real plane and noting that the QPSK performance will be identical. 
We can form the decision variable for subcarrier $k$ as

$$
\Re\left\{e^{-j \eta_{k}} r_{k}\right\}=\Re\left\{e^{-j \eta_{k}} \tilde{y}_{k}\right\}+\Re\left\{e^{-j \eta_{k}} \tilde{i}_{k}\right\}+\Re\left\{e^{-j \eta_{k}} \tilde{n}_{k}\right\} \triangleq y_{k}+i_{k}+n_{k}
$$

Since we have assumed BPSK transmission

$$
y_{k} \triangleq \Re\left\{e^{-j \eta_{k}} \tilde{y}_{k}\right\}=g_{k} x_{k}
$$

where $g_{k}=\Re\left\{e^{-j \eta_{k}} G_{k}\right\}$, while $n_{k} \triangleq \Re\left\{e^{-j \eta_{k}} \tilde{n}_{k}\right\}$ is AWGN and $i_{k} \triangleq \Re\left\{e^{-j \eta_{k}} \tilde{i}_{k}\right\}$ is given by

$$
\left.i_{k}=A \Re\left\{e^{j\left(\alpha-\eta_{k}\right)} \sum_{\ell=-\infty}^{\infty} \sum_{d=0}^{N_{\mathrm{n}}-1} z_{d, \ell} \beta_{k, \ell, d}\right\} \quad \text { (WiMAX }- \text { OFDM }\right)
$$

or

$$
i_{k}=A \Re\left\{e^{j\left(\alpha-\eta_{k}\right)} \sum_{\ell=-\infty}^{\infty} z_{\ell} \beta_{k, \ell}\right\} \quad(\text { WiMAX }-\mathrm{SC}) .
$$

For future reference, we define the signal-to-noise ratio (SNR) as

$$
\mathrm{SNR} \triangleq \frac{\mathbb{E}\left\{y_{k}^{2}\right\}}{\mathbb{E}\left\{2 n_{k}^{2}\right\}}=\frac{\mathbb{E}\left\{g_{k}^{2}\right\}}{2 \sigma_{n}^{2}}
$$

where $\sigma_{n}^{2}=\mathbb{E}\left\{n_{k}^{2}\right\}$ is the variance of $n_{k}$ (which is independent of $k$ ).

For subcarrier $k$, the signal-to-interference ratio $\left(\mathrm{SIR}_{k}\right)$ is given by

$$
\mathrm{SIR}_{k} \triangleq \frac{\mathbb{E}\left\{y_{k}^{2}\right\}}{\mathbb{E}\left\{2 i_{k}^{2}\right\}}=\frac{\mathbb{E}\left\{g_{k}^{2}\right\}}{2 \mathbb{E}\left\{A^{2}\right\} \sigma_{i, k}^{2}}
$$

where we have separated $\mathbb{E}\left\{A^{2}\right\}$ from $\sigma_{i, k}^{2}$ in order to account for possible random $A$, cf. Section 3.4, and $\sigma_{i, k}^{2}$ is given by

$$
\sigma_{i, k}^{2}=\frac{1}{2} \sum_{\ell=-\infty}^{\infty} \sum_{d=0}^{N_{\mathrm{n}}-1} \mathbb{E}\left\{\left|z_{d, \ell}\right|^{2}\right\}\left|\beta_{k, \ell, d}\right|^{2}=\frac{1}{2} \sum_{\ell=-\infty}^{\infty} \sum_{d=0}^{N_{\mathrm{n}}-1}\left|\beta_{k, \ell, d}\right|^{2} \quad(\text { WiMAX }- \text { OFDM })
$$

or

$$
\sigma_{i, k}^{2}=\frac{1}{2} \sum_{\ell=-\infty}^{\infty} \mathbb{E}\left\{\left|z_{\ell}\right|^{2}\right\}\left|\beta_{k, \ell}\right|^{2}=\frac{1}{2} \sum_{\ell=-\infty}^{\infty}\left|\beta_{k, \ell}\right|^{2} \quad \text { (WiMAX - SC) }
$$

where $\mathbb{E}\left\{\left|z_{d, \ell}\right|^{2}\right\}=1$ and $\mathbb{E}\left\{\left|z_{\ell}\right|^{2}\right\}=1$ since the transmitted symbols have unit energy. 
Given that the MB-OFDM system hops over three bands, but that the interference power in two of these bands is zero, the overall average SIR is given by

$$
\mathrm{SIR} \triangleq \frac{\mathbb{E}\left\{g_{k}^{2}\right\}}{2 \cdot \mathbb{E}\left\{A^{2}\right\} \cdot\left(\frac{1}{3 N_{\mathrm{m}}} \sum_{k=0}^{N_{\mathrm{m}}-1} \sigma_{i, k}^{2}\right)} .
$$

The symbols $x_{k}$ are equiprobable \pm 1 and $i_{k}$ and $n_{k}$ are zero mean and symmetric. Using properties of the Laplace transform [12], the probability of error for subcarrier $k$ is given by

$$
\begin{aligned}
P_{e, k} & =\operatorname{Prob}\left\{\left(i_{k}+n_{k}\right)<-g_{k}\right\}=\int_{-\infty}^{-g_{k}} p_{i_{k}+n_{k}}(x) \mathrm{d} x \\
& =\frac{1}{2 \pi j} \int_{c-j \infty}^{c+j \infty} \Phi_{i_{k}+n_{k}}(s) e^{-g_{k} s} \frac{\mathrm{d} s}{s}
\end{aligned}
$$

where $p_{i_{k}+n_{k}}(x)$ and $\Phi_{i_{k}+n_{k}}(s) \triangleq \mathbb{E}\left\{e^{-s\left(i_{k}+n_{k}\right)}\right\}$ denote the probability density function (pdf) of $\left(i_{k}+\right.$ $\left.n_{k}\right)$ and its Laplace transform, respectively, and $c$ is in the convergence region of $\Phi_{i_{k}+n_{k}}(s) e^{-g_{k} s} / s$. Due to the independence of $i_{k}$ and $n_{k}$,

$$
\Phi_{i_{k}+n_{k}}(s)=\Phi_{i_{k}}(s) \Phi_{n_{k}}(s)
$$

and since $n_{k}$ is Gaussian, its Laplace transform is [13]

$$
\Phi_{n_{k}}(s)=\exp \left(\frac{s^{2} \sigma_{n}^{2}}{2}\right)
$$

We are left with the determination of $\Phi_{i_{k}}(s)$. We begin by considering the conditional Laplace transform $\Phi_{i_{k} \mid \tau, \alpha}(s)=\mathbb{E}\left\{e^{-s i_{k}} \mid \tau, \alpha\right\}$, and treat the SC and OFDM cases separately.

1) WiMAX-OFDM: Since $z_{d, \ell}$ are independent, $\Phi_{i_{k} \mid \tau, \alpha}(s)$ is given by

$$
\Phi_{i_{k} \mid \tau, \alpha}(s)=\prod_{\ell=-\infty}^{\infty} \prod_{d=0}^{N_{\mathrm{n}}-1} \mathbb{E}\left\{\exp \left(-s \Re\left\{A e^{j\left(\alpha-\eta_{k}\right)} z_{d, \ell} \beta_{k, \ell, d}\right\}\right)\right\} .
$$

Depending on whether the WiMAX-OFDM symbols $z_{d, \ell}$ are chosen from the BPSK or the QPSK constellation, we arrive at

$$
\Phi_{i_{k} \mid \tau, \alpha}(s)=\prod_{\ell=-\infty}^{\infty} \prod_{d=0}^{N_{\mathrm{n}}-1} \cosh \left(s \Re\left\{A e^{j\left(\alpha-\eta_{k}\right)} \beta_{k, \ell, d}\right\}\right) \quad \text { (BPSK), }
$$


or

$$
\Phi_{i_{k} \mid \tau, \alpha}(s)=\prod_{\ell=-\infty}^{\infty} \prod_{d=0}^{N_{\mathrm{n}}-1} \cosh \left(s \Re\left\{A e^{j\left(\alpha-\eta_{k}\right)} \beta_{k, \ell, d}\right\}\right) \cosh \left(s \Im\left\{A e^{j\left(\alpha-\eta_{k}\right)} \beta_{k, \ell, d}\right\}\right) \quad \text { (QPSK). }
$$

2) WiMAX-SC: Since the $z_{\ell}$ are independent, $\Phi_{i_{k} \mid \tau, \alpha}(s)$ is given by

$$
\Phi_{i_{k} \mid \tau, \alpha}(s)=\prod_{\ell=-\infty}^{\infty} \mathbb{E}\left\{\exp \left(-s \Re\left\{A e^{j\left(\alpha-\eta_{k}\right)} z_{\ell} \beta_{k, \ell}\right\}\right)\right\} .
$$

Considering again the two choices of BPSK and QPSK for the symbols $z_{\ell}$ of the WiMAX-SC system, we arrive at

$$
\Phi_{i_{k} \mid \tau, \alpha}(s)=\prod_{\ell=-\infty}^{\infty} \cosh \left(s \Re\left\{A e^{j\left(\alpha-\eta_{k}\right)} \beta_{k, \ell}\right\}\right) \quad(\mathrm{BPSK})
$$

or

$$
\Phi_{i_{k} \mid \tau, \alpha}(s)=\prod_{\ell=-\infty}^{\infty} \cosh \left(s \Re\left\{A e^{j\left(\alpha-\eta_{k}\right)} \beta_{k, \ell}\right\}\right) \cosh \left(s \Im\left\{A e^{j\left(\alpha-\eta_{k}\right)} \beta_{k, \ell}\right\}\right) \quad \text { (QPSK) . }
$$

With the conditional Laplace transforms $\Phi_{i_{k} \mid \tau, \alpha}(s)$ given by (28)-(31), we can obtain the overall Laplace transform $\Phi_{i_{k}}(s)$. We let $\alpha^{\prime}=\alpha-\eta_{k}$, and note that it is uniformly distributed on $[0,2 \pi)$, so that $\Phi_{i_{k} \mid \tau, \alpha^{\prime}}(s)=\Phi_{i_{k} \mid \tau, \alpha}(s)$. By integrating over the distributions of $\alpha^{\prime}$ and $\tau$, we obtain $\Phi_{i_{k}}(s)$ as

$$
\Phi_{i_{k}}(s)=\frac{1}{2 \pi T_{X}} \int_{0}^{T_{X}} \int_{0}^{2 \pi} \Phi_{i_{k} \mid \tau, \alpha^{\prime}}(s) \mathrm{d} \alpha^{\prime} \mathrm{d} \tau
$$

Given (26) - (32), we can now determine the probability of error for subcarrier $k$, given by (25). Unfortunately, (25) does not have a closed-form solution and we must resort to numerical evaluation. This can be done efficiently via the Gauss-Chebyshev quadrature rule [12]

$$
P_{e, k} \approx \frac{1}{K} \sum_{\nu=1}^{K / 2}\left(\Re\left\{\Phi_{i_{k}+n_{k}}\left(c s_{\nu}\right) e^{-g_{k} c s_{\nu}}\right\}+\xi_{\nu} \Im\left\{\Phi_{i_{k}+n_{k}}\left(c s_{\nu}\right) e^{-g_{k} c s_{\nu}}\right\}\right),
$$

where $s_{\nu} \triangleq 1+j \xi_{\nu}, \xi_{\nu} \triangleq \tan ([2 \nu-1] \pi /[2 K])$, and $K$ is a sufficiently large integer. We have found a good choice is $K=200$ for the computations in Section 4. In general, the real-valued parameter $c$ should be chosen to minimize $\Phi_{i_{k}+n_{k}}(c) e^{-g_{k} c} / c$. We have found that a simpler yet suitable choice of $c$ is the value which minimizes $\left.\left(\Phi_{i_{k}+n_{k}}(c) e^{-g_{k} c} / c\right)\right|_{\tau=0, \alpha=0}$, which can very quickly be determined using standard numerical techniques. 


\subsection{Approximate BER with In-Band Interferer}

In this section we present an approximation of the BER calculated in Section 3.1. We make the assumption that the interference signal at subcarrier $k$ with power $A^{2} \sigma_{i, k}^{2}$ can be modeled as an additional zero-mean Gaussian noise signal with variance $A^{2} \sigma_{i, k}^{2}$, where $\sigma_{i, k}^{2}$ is defined in (22) or (23). In this case, the effective noise power is given by

$$
\sigma_{e, k}^{2}=\sigma_{n}^{2}+A^{2} \sigma_{i, k}^{2}
$$

and the BER for subcarrier $k$ is given by

$$
P_{a, k}=Q\left(\sqrt{g_{k}^{2} / \sigma_{e, k}^{2}}\right)
$$

\subsection{Overall BER Analysis for Non-Faded Channels}

In this section we consider the overall BER when $A=1$ and $g_{k}=1 \forall k$, i.e., we consider the case of non-faded channels for both the WiMAX and MB-OFDM signals. This case is of interest because it allows us to focus attention on the effect of the interference signal on the BER, while ignoring the contribution of fading.

When the WiMAX interferer is in the band of interest to the MB-OFDM system, the BER is given by (33) (exact) or (35) (approximate). On the other hand, when the MB-OFDM system hops to a different band, the interferer is not present and the BER is given by

$$
P_{n, k}=Q\left(\sqrt{g_{k}^{2} / \sigma_{n}^{2}}\right) .
$$

For first generation devices, the MB-OFDM hops over three bands with equal average usage, and the WiMAX system of interest is found in the first band. Noting that $P_{n}=P_{n, k}$ is independent of $k$ since $g_{k}=1 \forall k$, the overall BER is given by

$$
P=\frac{1}{3}\left(\frac{1}{N_{\mathrm{m}}} \sum_{k=0}^{N_{\mathrm{m}}-1} P_{Y, k}\right)+\frac{2}{3} P_{n},
$$

where $Y \in\{e, a\}$ depending on whether the exact or approximate BER expression is used for the band containing interference. 


\subsection{Overall BER Analysis for Faded Channels}

In the general case, $A$ and $g_{k}$ are distributed according to probability density functions $p_{A}(A)$ and $p_{g_{k}}\left(g_{k}\right)$, respectively. In order to obtain the overall average BER in the presence of fading, we average (25), (35), and (36) over these densities.

We first consider (25), and take first the expectation over $g_{k}$

$$
\mathbb{E}_{g_{k}}\left\{P_{e, k}\right\}=\frac{1}{2 \pi j} \int_{c-j \infty}^{c+j \infty} \Phi_{i_{k}+n_{k}}(s) \mathbb{E}_{g_{k}}\left\{e^{-g_{k} s}\right\} \frac{\mathrm{d} s}{s}=\frac{1}{2 \pi j} \int_{c-j \infty}^{c+j \infty} \Phi_{i_{k}+n_{k}}(s) \Phi_{g_{k}}(s) \frac{\mathrm{d} s}{s},
$$

where $\Phi_{g_{k}}(s)$ is the Laplace transform of the pdf of $g_{k}$. We note that (38) can again be evaluated using the Gauss-Chebyshev quadrature rule [12], cf. (33). The average exact BER in the presence of in-band interference is then given by

$$
\bar{P}_{e, k}=\int_{0}^{\infty} \frac{1}{2 \pi j} \int_{c-j \infty}^{c+j \infty} \Phi_{i_{k}+n_{k}}(s) \Phi_{g_{k}}(s) \frac{\mathrm{d} s}{s} p_{A}(A) \mathrm{d} A .
$$

We turn to the consideration of (35). We first take $\mathbb{E}_{g_{k}}\left\{P_{a, k}\right\}$, which, by using an alternative form of the Q-function [14], can be written as

$$
\mathbb{E}_{g_{k}}\left\{P_{a, k}\right\}=\int_{0}^{\infty} Q\left(\sqrt{\frac{\gamma_{k}}{\sigma_{e, k}^{2}}}\right) p_{\gamma_{k}}\left(\gamma_{k}\right) \mathrm{d} \gamma_{k}=\frac{1}{\pi} \int_{0}^{\pi / 2} M_{\gamma_{k}}\left(\frac{-1}{2\left(\sigma_{n}^{2}+A^{2} \sigma_{i, k}^{2}\right) \sin ^{2} \lambda}\right) \mathrm{d} \lambda,
$$

where $\gamma_{k}=g_{k}^{2}, p_{\gamma_{k}}\left(\gamma_{k}\right)$ is the pdf of $\gamma_{k}$, and $M_{\gamma_{k}}(s)=\mathbb{E}\left\{e^{s \gamma_{k}}\right\}$ is the moment generating function of $\gamma_{k}$ [14]. We can then express the average approximate BER in the presence of in-band interference as

$$
\bar{P}_{a, k}=\frac{1}{\pi} \int_{0}^{\infty} \int_{0}^{\pi / 2} M_{\gamma_{k}}\left(\frac{-1}{2\left(\sigma_{n}^{2}+A^{2} \sigma_{i, k}^{2}\right) \sin ^{2} \lambda}\right) \mathrm{d} \lambda p_{A}(A) \mathrm{d} A .
$$

Using similar techniques as with (40), we can express the average BER without interference as [14]

$$
\bar{P}_{n, k}=\frac{1}{\pi} \int_{0}^{\pi / 2} M_{\gamma_{k}}\left(\frac{-1}{2 \sigma_{n}^{2} \sin ^{2} \lambda}\right) \mathrm{d} \lambda
$$


Finally, the overall average BER is given by

$$
\bar{P}=\frac{1}{3}\left(\frac{1}{N_{\mathrm{m}}} \sum_{k=0}^{N_{\mathrm{m}}-1} \bar{P}_{Y, k}\right)+\frac{2}{3}\left(\frac{1}{N_{\mathrm{m}}} \sum_{k=0}^{N_{\mathrm{m}}-1} \bar{P}_{n, k}\right),
$$

with $Y \in\{e, a\}$ depending on whether (39) or (41) is used. Note that if $p_{g_{k}}\left(g_{k}\right)$ is independent of $k$, then the second term in (43) can be simplified as was done in (37). We note that the integrals with semi-infinite limits in (39) and (41) converge quite rapidly and can be truncated by using a finite upper limit without loss of accuracy.

\section{Results for Uncoded MB-OFDM}

In this section, we (a) investigate the effect of WiMAX interference on MB-OFDM systems, and (b) study the applicability of the Gaussian approximation for WiMAX interference. The latter is especially important for the design of interference mitigation schemes, and for simplified performance analysis.

\subsection{WiMAX-OFDM Interference}

Figure 2 shows the BER versus $10 \log _{10}(\mathrm{SIR})$ from exact analysis (lines) and the Gaussian approximation (markers) for BPSK/QPSK WiMAX-OFDM interference of varying bandwidth and for different SNR. The results for BPSK and QPSK are virtually identical, so we have only included the BPSK results in this figure. In order to isolate the effects of the interference signal, we have chosen to fix $A=1$ and $g_{k}=1 \forall k$, i.e., we consider the case of non-faded channels for both the WiMAX and MB-OFDM signals.

We can see that the Gaussian approximation is an excellent match with the exact analysis for WiMAX-OFDM interference, for all considered values of SNR, SIR, and WiMAX bandwidths. This can be justified intuitively, since all subcarriers of the WiMAX-OFDM signal contribute to each time-domain sample of the interference signal, and thus there is a natural averaging / Central Limit Theorem effect. We note that in [9], the authors found that a Gaussian approximation was not appropriate for BPSK-modulated narrowband OFDM in some ranges of interest. However, this 
trend is not evidenced here, likely because WiMAX-OFDM employs $N_{\mathrm{n}}=256$ subcarriers versus the relatively smaller $N_{\mathrm{n}}=64$ of [9], which increases the averaging effect and hence the Gaussianity of the interference. We also note that, for a fixed $10 \log _{10}(\mathrm{SIR})$, the BER tends to decrease as the interferer bandwidth increases. This is because the per-subcarrier interference power decreases as the bandwidth increases (since the average interference power is constant), and thus (since the BER decays exponentially with increasing $\mathrm{SIR}_{k}$ ) the values of $P_{e, k}$ also decrease with increasing interference bandwidth.

To confirm the results of the analysis, Figure 3 shows the BER versus $10 \log _{10}(\mathrm{SIR})$ for both the exact analysis (lines) and simulation results (markers), with non-faded channels for both the QPSK WiMAX and MB-OFDM signals. We note an excellent agreement between analysis and simulation for all considered parameters.

Finally, we consider Rayleigh distributed amplitudes $g_{k}$ (a good approximation for UWB channels $[15,16]$ ), with $A=1$ (corresponding to a WiMAX transmitter in close proximity to the MB-OFDM receiver). Figure 4 shows the BER versus $10 \log _{10}(\mathrm{SIR})$ from both the exact analysis (lines) and the Gaussian approximation (markers). The Gaussian approximation is still an excellent match with the exact analysis. Fading in the MB-OFDM channel causes fluctuations in the instantaneous signal-to-interference ratio, which in turn decreases the distinction between different WiMAX-OFDM bandwidths at moderate to high SIR. The same fluctuations also increase the average SIR required in order to approach the interference-free error rate.

\subsection{WiMAX-SC Interference}

In this section, we present numerical results illustrating the performance analysis methods applied to WiMAX-SC interference. We concentrate on the case of $A=1$ and $g_{k}=1 \forall k$, i.e., the case of non-fading channels for both the MB-OFDM and WiMAX-SC transmissions. The results below have also been confirmed via simulations, which we have omitted for clarity. 
We first consider WiMAX-SC with BPSK modulation. In Figure 5 we plot the BER versus $10 \log _{10}(\mathrm{SIR})$ for different WiMAX-SC bandwidths, for $10 \log _{10}(\mathrm{SNR})=10$. We show both the

exact analysis (lines) and the Gaussian approximation (markers). The Gaussian approximation is very accurate for small and large SIR, with some deviation at intermediate values of SIR. We can also see that the Gaussian approximation is worst for small WiMAX-SC bandwidths, and improves as the bandwidth increases. This is due to the shorter symbol time of the wide bandwidth WiMAX-SC signal, leading to a more pronounced averaging effect of the interference during one MB-OFDM symbol duration.

In Figure 6 we consider QPSK WiMAX modulation, and plot the BER versus $10 \log _{10}(\mathrm{SIR})$ for different WiMAX bandwidths. We can see the Gaussian approximation is improved in comparison with Figure 5, due to the increased randomness of the four-phase QPSK signal. We expect the accuracy of the Gaussian approximation to continue to improve for higher-order QAM modulations.

\section{Interference Mitigation for Coded MB-OFDM}

It is natural to seek means to mitigate the impact of WiMAX interference on MB-OFDM systems. The results of the previous section have shown that the MB-OFDM per-subcarrier interferenceplus-noise distribution in the presence of WiMAX interference behaves in an approximately Gaussian manner. Given the near-Gaussian nature of these per-subcarrier interference statistics, one natural and near-optimum technique for interference mitigation is to (1) estimate the per-subcarrier interference-plus-noise power, and (2) use this information to weigh the branch metrics fed to the Viterbi decoder, in order to suppress the interference effects. This particular technique requires only modest increases in receiver complexity, and does not require any modifications to the MB-OFDM transmitter or signal structure. We describe each stage of the process below. 


\subsection{Interference Estimation}

The MB-OFDM system will listen to the channel for interference estimation purposes, either (a) during the silent period between packet transmissions, or by (b) listening to one sub-band while operating on another sub-band. Furthermore, we assume that no other UWB devices transmit in the considered sub-band during the silent time, so that the receiver will detect only the interferenceplus-noise that exists in the channel. We let $P$ be the number of MB-OFDM symbol durations that are used to observe each sub-band.

We consider two methods for spectral estimation, described below. Both approaches adopt a time-domain estimation followed by a Fourier transform to obtain the final per-subcarrier noise variance estimates. Time-domain estimation allows us to exploit the limited degrees of freedom in the interference signal. We denote the resultant interference-plus-noise variance estimate for subcarrier $k$ by $\hat{S}\left(\omega_{k}\right)$, where $\omega_{k}=2 \pi k / N_{\mathrm{m}}$.

\subsubsection{Parametric Approach}

We first adopt a parametric approach by fitting the time-domain silent period observations to an autoregressive (AR) model. The fitting method is that of maximum entropy, also known as the Burg method [17]. For a given AR model order $M$, the Burg spectral estimate is given by

$$
\hat{S}_{\text {Burg }}(\omega)=\frac{P_{M}}{\left|1+\sum_{i=1}^{M} a_{M, i} e^{-j i \omega}\right|^{2}},
$$

where $P_{M}$ and $a_{M, i}$ are the parameters of the AR model, obtained with the Levinson-Durbin algorithm [17, Sec. 9.5, pp. 414-420]. The parametric approach is generally able to use small estimation periods $P$. However, the performance of the method is dependent on a proper choice of model order $M$ - smaller model orders lead to faster estimation, but may not yield suitable estimates in the presence of multiple interferers or other complicated interference scenarios. 


\subsubsection{Non-parametric Approach}

We also consider a non-parametric approach to spectral estimation. We adopt the multi-taper method (MTM) [17], advocated for use in radio-scene analysis for cognitive radio [18]. In the MTM, a set of orthogonal windows (or tapers) $w_{i}(n)$ are applied to the observed data and the resultant estimates are averaged. In this work we adopt tapers based on the Slepian (or discrete prolate spheroidal) sequences [17, Chap. 8], which have maximal energy concentration for finite bandwidth and sample size. We use $P$ tapers for a window of $P$ MB-OFDM observation symbols, for a total of $N=(128+37) P$ samples taken at rate $T=1 /\left(528 \times 10^{6}\right) \mathrm{s}$. The MTM spectral estimate of the discrete-time observed signal $b(n T)$ is given by

$$
\hat{S}_{\mathrm{MTM}}(\omega)=\frac{1}{P} \sum_{i=0}^{P-1}\left|\sum_{n=0}^{N-1} w_{i}(n) b(n T) e^{-j \omega n T}\right|^{2} .
$$

\subsection{Interference Mitigation}

The estimators given above predict the interference seen at the input to the MB-OFDM receiver FFT. However, we will mitigate the interference during decoding, after the FFT, and thus must account for the effect of the rectangular time-domain window of length $D_{\mathrm{m}}$. The spectrum after windowing is given by $\hat{S}_{W}(\omega)=\hat{S}_{X}(\omega) \otimes\left[\frac{\sin \left(\omega D_{\mathrm{m}}\right)}{\omega}\right]^{2}$, with $X \in\{\operatorname{Burg}, \mathrm{MTM}\}$.

The MB-OFDM system employs bit-interleaved coded modulation (BICM) for error protection [19]. Decoding consists of soft de-mapping, followed by de-interleaving and Viterbi decoding. Given that the interference-plus-noise per subcarrier is approximately Gaussian, we maintain the standard Euclidean distance metric, and scale the branch metrics for all bits from subcarrier $k$ by $1 / \hat{S}_{W}\left(\omega_{k}\right)$. The effect of the correlation between adjacent subcarriers is negligible due to the de-interleaving process, and can be neglected.

Note that, in the presence of purely Gaussian noise, the strategy described above is optimal. We do not simply discard information from subcarriers, but rather reduce the influence of bits which have been impacted by WiMAX interference. We note that erasure decoding (as proposed in e.g. [20]) 
can be seen as a special case of this technique when $\hat{S}_{W}\left(\omega_{k}\right) \rightarrow \infty$ for some $k$.

\section{Results for Coded MB-OFDM}

In this section we present results for coded MB-OFDM systems employing the interference estimation and mitigation technique discussed in Section 5. We focus on the case of WiMAX-OFDM interference in order to illustrate the potential performance gains of this technique. Because we want to isolate the effects of the interference mitigation, we focus on non-fading WiMAX channels, and note that similar behaviors will be observed with fading channels. We also assume the WiMAX system is continually transmitting, i.e., we do not consider arrival/departure of WiMAX systems during the transmission interval. Interference estimation is performed anew before each MB-OFDM data packet transmission, and the estimates are then fixed for the duration of the MB-OFDM packet.

In Figure 7, we plot the MB-OFDM BER versus $10 \log _{10}(\mathrm{SIR})$ for coded transmission with rate $1 / 2$ and $10 \log _{10}(\mathrm{SNR})=14.5$. For the MB-OFDM system, we adopt the UWB channel model CM1 [15], and average over 500 channel realizations. The interferer is a QPSK WiMAX-OFDM system with bandwidth $7 \mathrm{MHz}$ and carrier frequency $f_{n}=3500 \mathrm{MHz}$. We consider both the Burg with $M=8$ (solid lines) and MTM (dashed lines) spectral estimation techniques, for $P \in\{2,5,50\}$ symbols. For comparison we also include the MB-OFDM BER with no spectral estimation, perfect spectral estimation, and no interference (thick dash-dotted lines). The perfect spectral estimation curves are obtained by assuming the receiver has perfect knowledge of the noise variance $\sigma_{n}^{2}$ and interference variances $\sigma_{i, k}^{2}$ (given by $(22)$ ) when calculating the branch metric weights as described in Section 5.2.

We make several observations about the results in Figure 7. Firstly, for small numbers of estimation symbols $P \in\{2,5\}$ the MTM estimation technique performs poorly, because such small observation lengths are not sufficient to provide reliable non-parametric estimation. On the other hand, for $P=50$ observation symbols the MTM method is comparable to the parametric approach. Secondly, we observe that for low values of SIR, the Burg estimator performance is relatively invariant 
to the choice of $P$, while at higher SIR there are slight gains with increasing $P$. Finally, we note that both the parametric (with $P=50$ ) and non-parametric approaches perform relatively close to the perfect estimation limit, and also provide substantial performance improvements in comparison with the case of no interference mitigation.

In Figure 8, we examine the effect of varying the Burg AR model order $M$. We adopt a nonfading MB-OFDM channel, code rate $1 / 2,10 \log _{10}(\mathrm{SNR})=4.1$, one WiMAX-OFDM interferer with bandwidth 1.75 $\mathrm{MHz}, \mathrm{AR}$ model orders $M \in\{4,8,16,32\}$ and $P \in\{2,10,50\}$ estimation symbols. We can see that at low SIR all model orders have relatively similar performance, with $M=4$ providing slightly better performance than for larger $M$. At higher SIR, increased model orders lead to better performance for $P=50$, but poor performance for the short estimation interval $P=2$. At intermediate values of SIR, model order $M=4$ is insufficient, with inaccurate modeling of the interference spectrum leading to degrading performance with increasing SIR. These results indicate the importance of choosing appropriate estimation parameters $M$ and $P$ in order to guarantee reasonable interference mitigation performance.

We consider a more complicated interference scenario in Figure 9, with two WiMAX-OFDM systems operating at $3475 \mathrm{MHz}$ and $3500 \mathrm{MHz}$, each with a bandwidth of $7 \mathrm{MHz}$. The MB-OFDM system operates over UWB channel CM1. We also consider a higher code rate of $3 / 4$, which provides less error protection to the transmitted MB-OFDM data. We can see that in this case, the Burg spectral estimator with $M=8$ does not perform well in the intermediate SIR range, due to the inability of the $M=8$ tap AR model to accurately represent the interference spectrum. On the other hand, both the MTM and Burg with $M=16$ perform well for all values of SIR. This result indicates that some consideration of the potential interference environment must be made in the design of interference mitigation techniques for MB-OFDM systems. In general, if larger values of $P$ can be tolerated, the MTM estimator may be preferable, while for smaller $P$ a Burg estimator with properly selected $M$ offers reasonable performance. 


\section{Conclusions}

Coexistence and the ability to appropriately handle interference from incumbent narrowband systems are important aspects of the design of UWB devices. The particular example of WiMAX in the 3.5 $\mathrm{GHz}$ band is of practical interest due to the potential for large-scale WiMAX deployment in the near future.

In this paper, we have presented both exact (using Laplace transform techniques) and approximate analysis of the BER of MB-OFDM in the presence of WiMAX interference. The two analysis methods are in excellent agreement, and furthermore are corroborated by simulation results. We have also shown via BER comparisons that the WiMAX interference has an approximately Gaussian behaviour on a per-subcarrier basis.

Motivated by the approximately Gaussian nature of the interference, we have presented a twostage interference mitigation technique, consisting of interference spectral estimation followed by interference mitigation during Viterbi decoding. We have compared parametric and non-parametric approaches for several interference scenarios of practical interest. In the presence of WiMAX interference, the two-stage interference mitigation provides substantial gains in performance in return for modest increases in receiver complexity and without requiring any modifications to the MBOFDM transmitter or signal structure. However, our results show that the expected interference environment should be carefully considered during the design of such mitigation techniques.

\section{References}

[1] ECMA, "Standard ECMA-368: High Rate Ultra Wideband PHY and MAC Standard," Dec. 2005, [Online]: http://www.ecma-international.org/publications/standards/Ecma-368.htm.

[2] A. Batra, J. Balakrishnan, G. Aiello, J. Foerster, and A. Dabak, "Design of a Multiband OFDM System for Realistic UWB Channel Environments," IEEE Trans. Microwave Theory Tech., vol. 52, no. 9, pp. 2123-2138, Sept. 2004.

[3] Federal Communications Commission (FCC), "Revision of Part 15 of the Commissions Rules Regarding Ultra-Wideband Transmission Systems," First Report and Order, ET Docket 98-153, FCC 02-48; Adopted: February 14, 2002; Released: April 22, 2002.

[4] Q. Zhao and B. M. Sadler, "A Survey of Dynamic Spectrum Access," IEEE Signal Processing Mag., vol. 24, no. 3, pp. 79-89, May 2007. 
[5] IEEE Std 802.16-2004, "Part 16: Air Interface for Fixed Broadband Wireless Access Systems," Oct. 2004.

[6] V. Somayazulu, J. Foerster, and R. Roberts, "Detect and Avoid (DAA) Mechanisms for UWB Interference Mitigation," in Proc. IEEE Intl. Conf. on Ultra-Wideband (ICUWB), Waltham, MA, USA, Sept. 2006, pp. 513-518.

[7] A. Durantini, R. Giuliano, F. Mazzenga, and F. Vatalaro, "Performance Evaluation of Detect and Avoid Procedures for Improving UWB Coexistence with UMTS and WiMAX systems," in Proc. IEEE Intl. Conf. on Ultra-Wideband (ICUWB), Waltham, MA, USA, Sept. 2006, pp. 501-506.

[8] K. Shi, Y. Zhou, B. Kelleci, T. W. Fischer, E. Serpedin, and A. I. Karșilayan, "Impacts of Narrowband Interference on OFDM-UWB Receivers: Analysis and Mitigation," IEEE Trans. Signal Processing, vol. 55, no. 3, pp. 1118-1128, Mar. 2007.

[9] B. Hu and N. C. Beaulieu, "Performance of an Ultra-Wideband Communication System in the Presence of Narrowband BPSK- and QPSK-Modulated OFDM Interference," IEEE Trans. Commun., vol. 54, no. 10, pp. 1720-1724, Oct. 2006.

[10] J. G. Proakis, Digital Communications, 4th ed. McGraw-Hill, 2001.

[11] O. Edfors, M. Sandell, J.-J. van de Beek, D. Landström, and F. Sjöberg, "An Introduction to Orthogonal Frequency-Division Multiplexing," [Online]: http://www.sm.luth.se/csee/sp/research/report/esb96rc.pdf, 1996.

[12] E. Biglieri, G. Caire, G. Taricco, and J. Ventura-Traveset, "Computing Error Probabilities over Fading Channels: a Unified Approach," European Transactions on Telecommunications, vol. 9, pp. 15-25, January/February 1998.

[13] A. Papoulis, Probability, Random Variables, and Stochastic Processes, 2nd ed. McGraw-Hill, 1984.

[14] M. K. Simon and M.-S. Alouini, Digital Communication over Fading Channels, 2nd ed. Wiley, 2005.

[15] A. F. Molisch, J. R. Foerster, and M. Pendergrass, "Channel Models for Ultrawideband Personal Area Networks," IEEE Wireless Commun. Mag., pp. 14-21, Dec. 2003.

[16] C. Snow, L. Lampe, and R. Schober, "Performance Analysis and Enhancement of Multiband OFDM for UWB Communications," IEEE Trans. Wireless Commun., vol. 6, no. 6, pp. 21822192, June 2007.

[17] D. B. Percival and A. T. Walden, Spectral Analysis for Physical Applications: Multitaper and Conventional Univariate Techniques. Cambridge University Press, 1993.

[18] S. Haykin, "Cognitive Radio: Brain-Empowered Wireless Communications," IEEE J. Select. Areas Commun., vol. 23, no. 2, pp. 201-220, Feb. 2005.

[19] G. Caire, G. Taricco, and E. Biglieri, "Bit-Interleaved Coded Modulation," IEEE Trans. Inform. Theory, vol. 44, no. 3, pp. 927-946, May 1998.

[20] T. Li, W. H. Mow, V. K. Lau, M. Siu, R. S. Cheng, and R. D. Murch, "Robust Joint Interference Detection and Decoding for OFDM-Based Cognitive Radio Systems with Unknown Interference," IEEE J. Select. Areas Commun., vol. 25, no. 3, pp. 566-575, Apr. 2007. 


\section{Figures and Tables}

Table 1: Relevant system parameters.

\begin{tabular}{|l|l|}
\hline Parameter & Value \\
\hline \hline \multicolumn{2}{|c|}{ MB-OFDM [1,2] } \\
\hline$N_{\mathrm{m}}$ & $128 \mathrm{subcarriers}$ \\
\hline$W_{\mathrm{m}}$ & $528 \mathrm{MHz}$ \\
\hline$T_{\mathrm{m}}$ & $312.5 \mathrm{~ns}$ \\
\hline$C_{\mathrm{m}}$ & $70.07 \mathrm{~ns}$ \\
\hline$D_{\mathrm{m}}$ & $242.43 \mathrm{~ns}$ \\
\hline$Q_{\mathrm{m}}$ & $4.125 \mathrm{MHz}$ \\
\hline \multicolumn{1}{|c|}{ WiMAX-OFDM $[5]$} \\
\hline$N_{\mathrm{n}}$ & 256 subcarriers \\
\hline$W_{\mathrm{n}}$ & $\begin{array}{l}\{2,4,6,8,20\} \mathrm{MHz}(\text { actual) } \\
\{1.75,3.5,5.25,7,17.5\} \mathrm{MHz}(\text { nominal) }\end{array}$ \\
\hline$T_{\mathrm{n}}$ & $1.25 N_{\mathrm{n}} / W_{\mathrm{n}}$ \\
\hline$C_{\mathrm{n}}$ & $0.25 N_{\mathrm{n}} / W_{\mathrm{n}}$ \\
\hline$D_{\mathrm{n}}$ & $T_{\mathrm{n}}-C_{\mathrm{n}}=N_{\mathrm{n}} / W_{\mathrm{n}}$ \\
\hline$Q_{\mathrm{n}}$ & $W_{\mathrm{n}} / N_{\mathrm{n}}$ \\
\multicolumn{2}{|c|}{ WiMAX-SC $[5]$} \\
\hline$T_{\mathrm{s}}$ & $\begin{array}{l}\{847.74,411.45,202.86,100.71,50.177,25.044\} \mathrm{ns} \\
\text { for bandwidths of }\{1.5625,3.125,6.25,12.5,25,50\} \mathrm{MHz}\end{array}$ \\
\hline
\end{tabular}




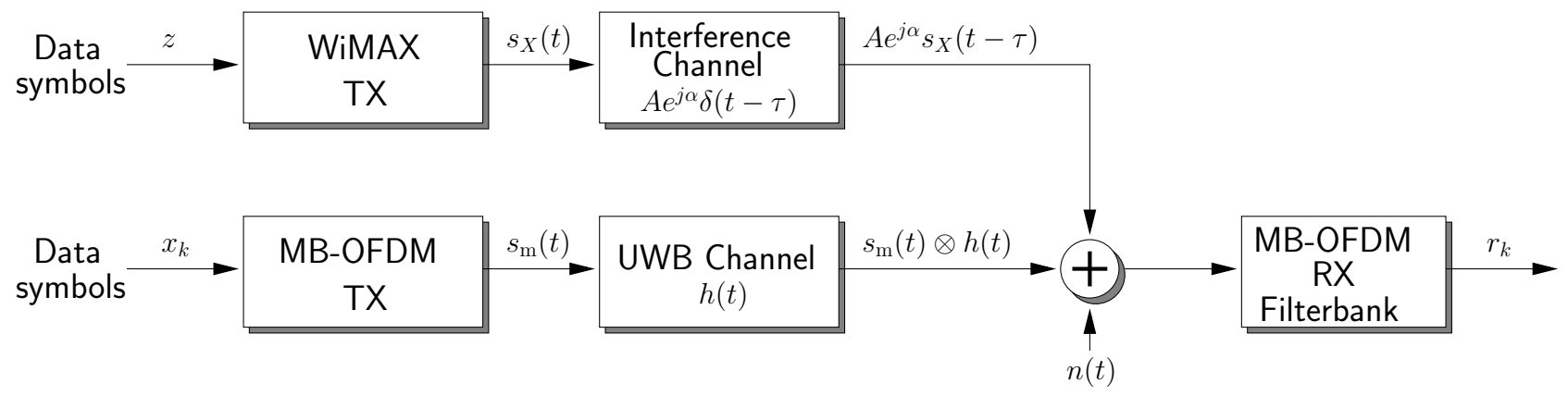

Figure 1: System model. $X \in\{\mathrm{n}, \mathrm{s}\}$ for WiMAX-OFDM and WiMAX-SC, respectively. 


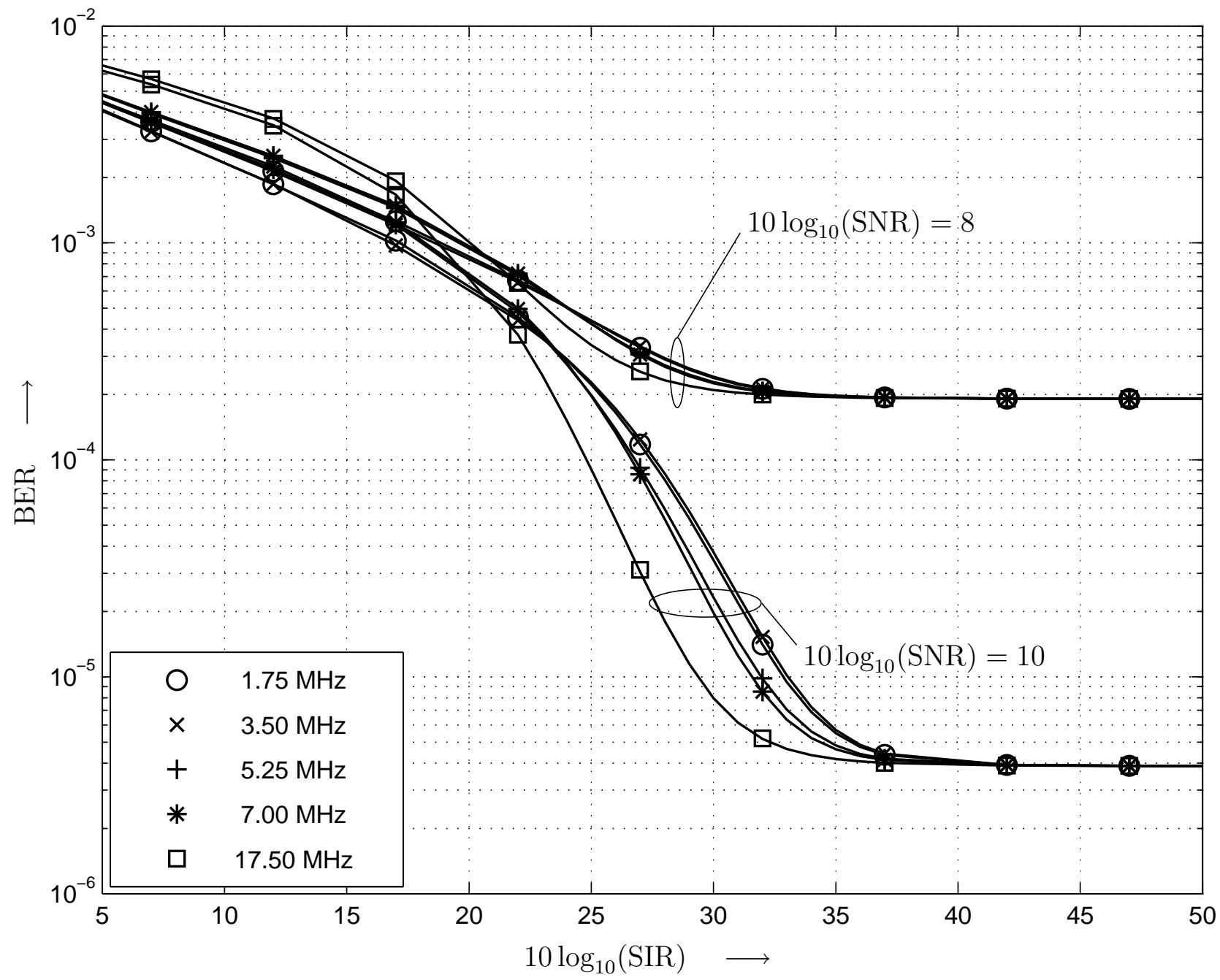

Figure 2: BER versus $10 \log _{10}(\mathrm{SIR})$ from exact analysis (lines) and Gaussian approximation (markers) for $10 \log _{10}(\mathrm{SNR}) \in\{8,10\}$ and WiMAX-OFDM bandwidths of $\{1.75,3.5,5.25,7,17.5\} \mathrm{MHz}$. BPSK WiMAX-OFDM, carrier frequency $f_{\mathrm{n}}=3500 \mathrm{MHz}$. $A=1$ and $g_{k}=1 \forall k$. 


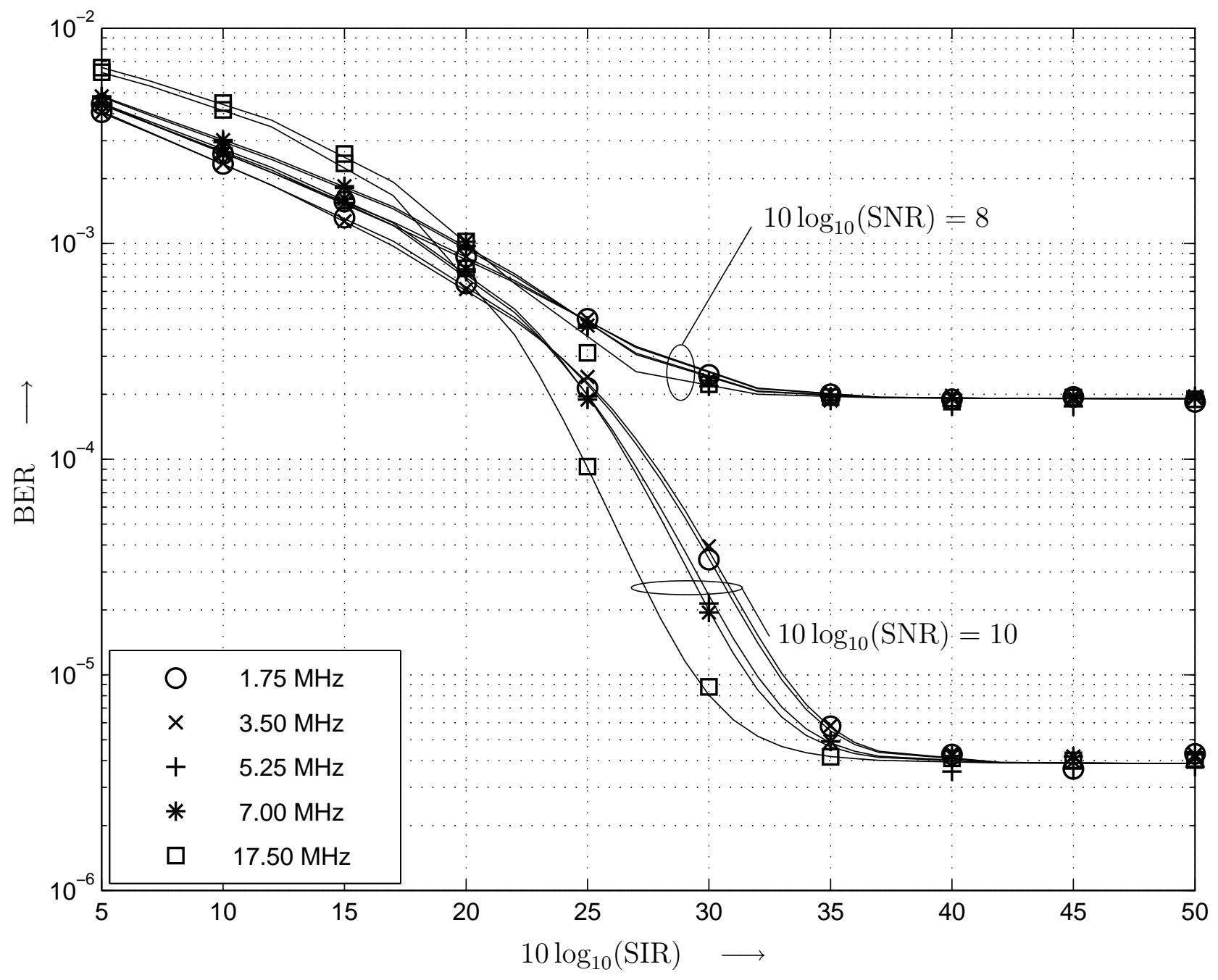

Figure 3: BER versus $10 \log _{10}(\mathrm{SIR})$ from exact analysis (lines) and simulation (markers) for $10 \log _{10}(\mathrm{SNR}) \in\{8,10\}$ and WiMAX-OFDM bandwidths of $\{1.75,3.5,5.25,7,17.5\} \mathrm{MHz}$. QPSK WiMAX-OFDM, carrier frequency $f_{\mathrm{n}}=3500 \mathrm{MHz}$. $A=1$ and $g_{k}=1 \forall k$. 


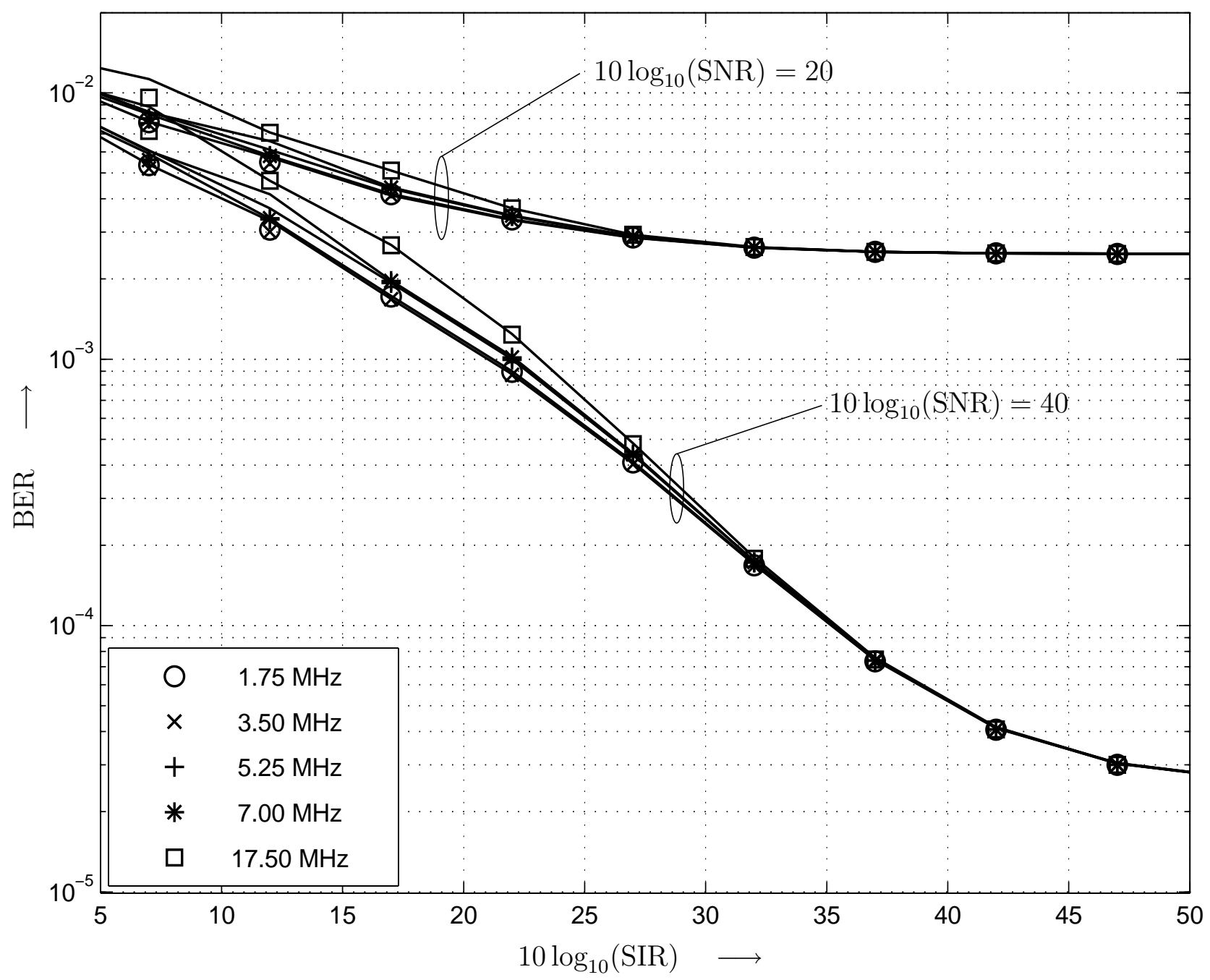

Figure 4: BER versus $10 \log _{10}$ (SIR) from exact analysis (lines) and Gaussian approximation (markers) for $10 \log _{10}(\mathrm{SNR}) \in\{20,40\}$ and WiMAX-OFDM bandwidths of $\{1.75,3.5,5.25,7,17.5\} \mathrm{MHz}$. QPSK WiMAX-OFDM, carrier frequency $f_{\mathrm{n}}=3500 \mathrm{MHz} . A=1, g_{k}$ Rayleigh. 


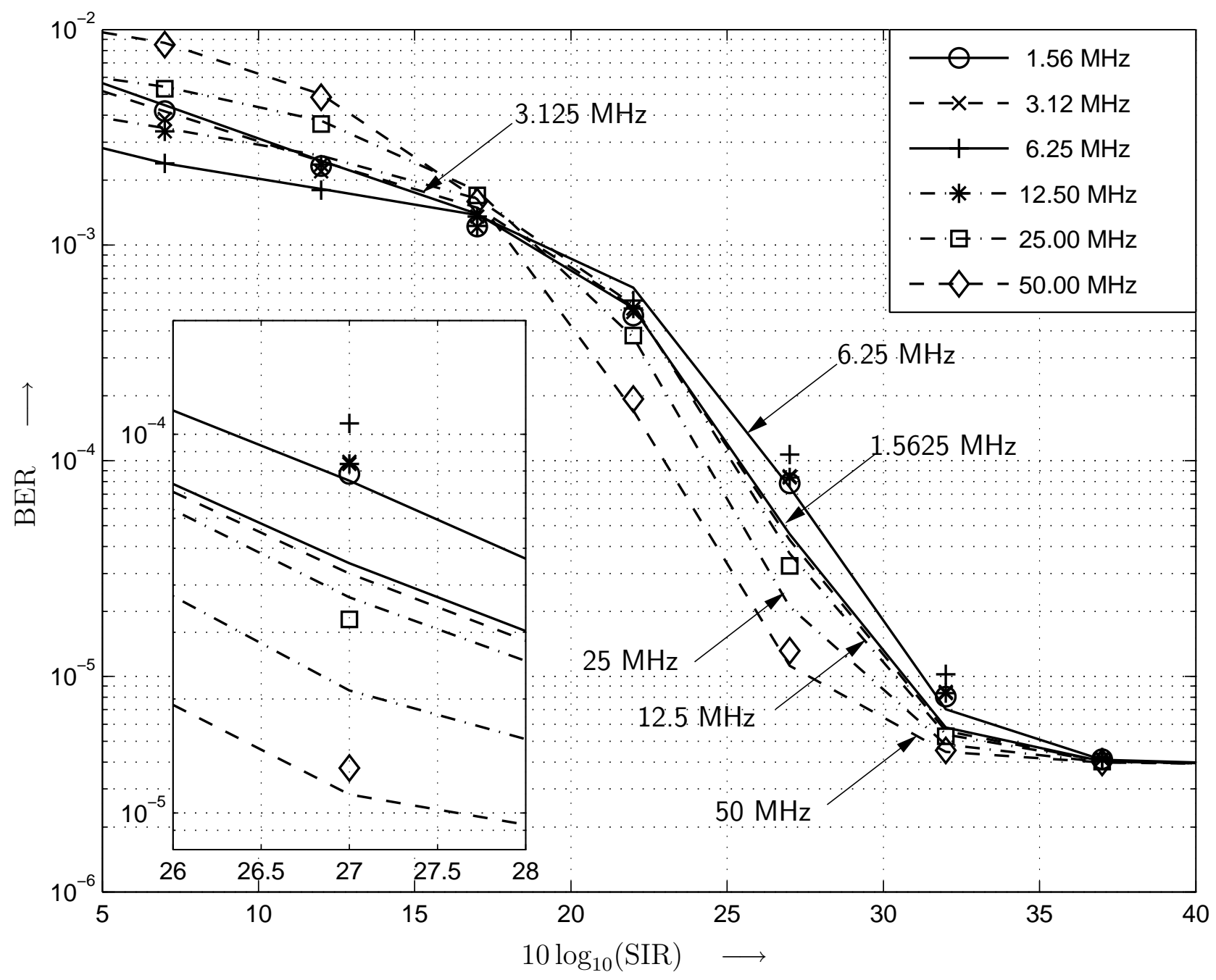

Figure 5: BER versus $10 \log _{10}(\mathrm{SIR})$ for $10 \log _{10}(\mathrm{SNR})=10$ and various WiMAX-SC bandwidths, with exact analysis (lines) and Gaussian approximation (markers). Inset: Zoomed version of same figure, showing difference between Gaussian approximation and exact BER. BPSK WiMAX-SC modulation. 


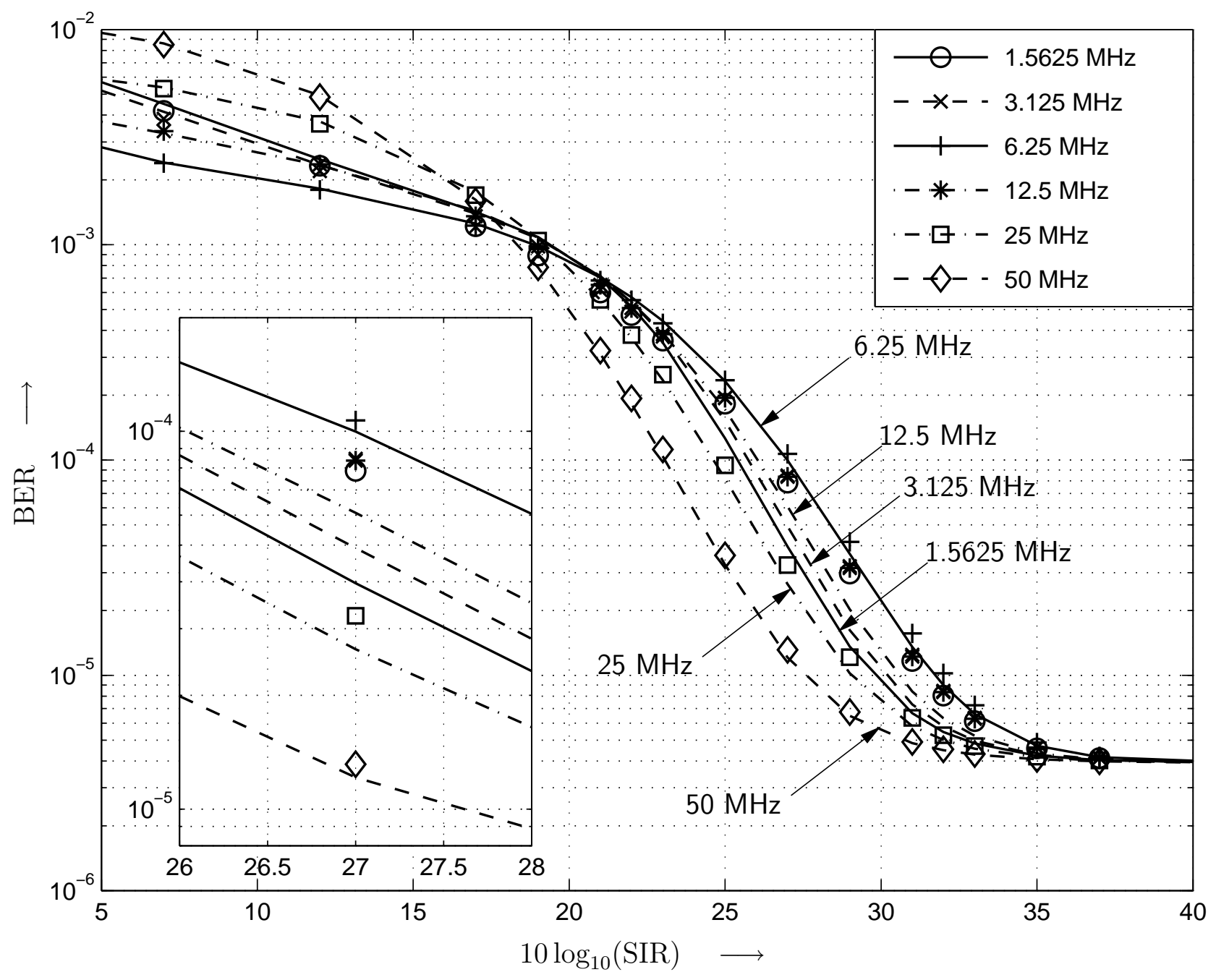

Figure 6: BER versus $10 \log _{10}(\mathrm{SIR})$ for $10 \log _{10}(\mathrm{SNR})=10$ and various WiMAX-SC bandwidths, with exact analysis (lines) and Gaussian approximation (markers). Inset: Zoomed version of same figure, showing difference between Gaussian approximation and exact BER. QPSK WiMAX-SC modulation. 


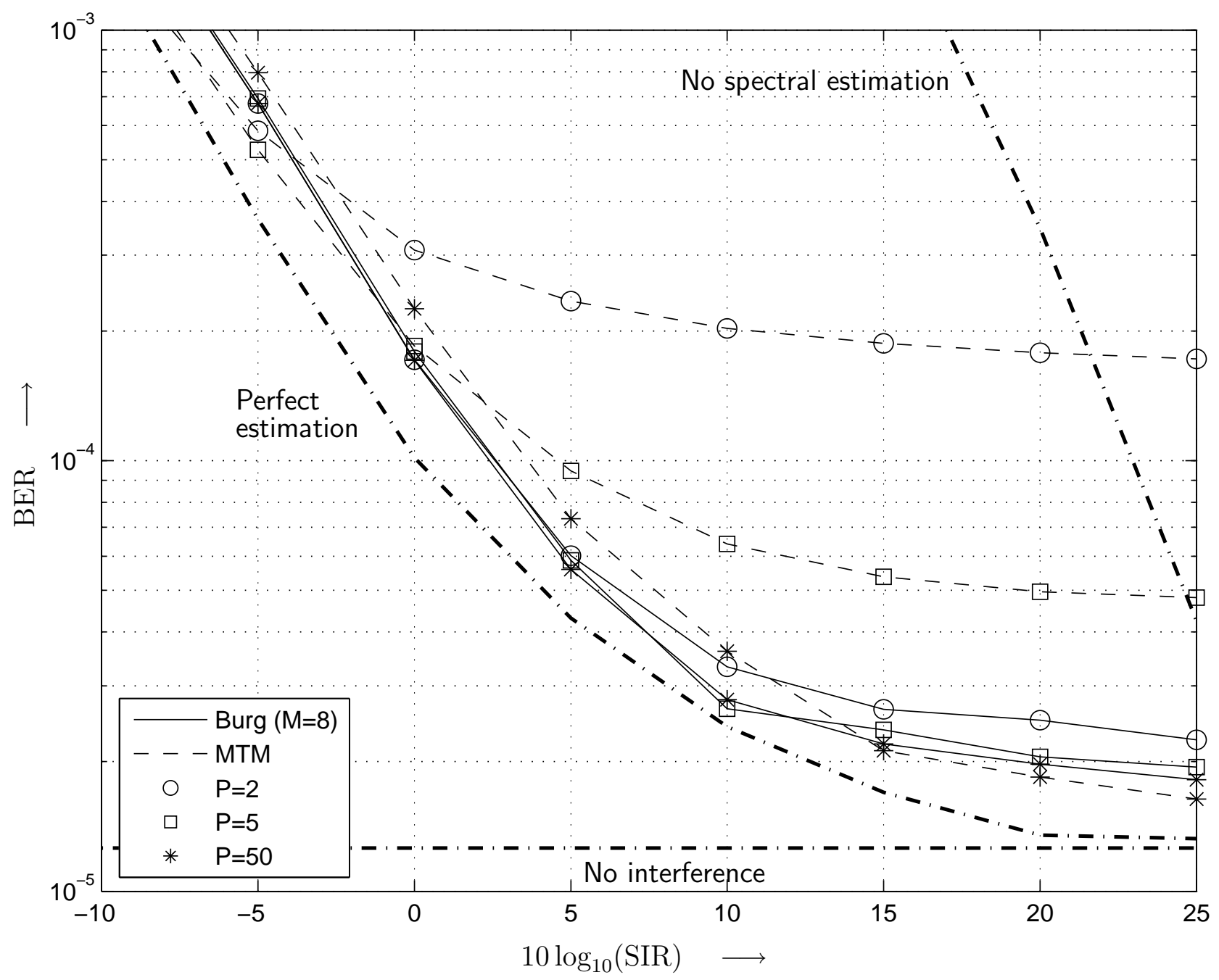

Figure 7: BER versus $10 \log _{10}(\mathrm{SIR})$ for $10 \log _{10}(\mathrm{SNR})=14.5$. MB-OFDM coded transmission with rate $1 / 2$. QPSK WiMAX-OFDM bandwidth $7 \mathrm{MHz}$, carrier frequency $f_{n}=3500 \mathrm{MHz}$. UWB CM1 channel, non-fading WiMAX channel. Burg with $M=8$ (solid lines) and MTM (dashed lines) spectral estimation techniques, for $P \in\{2,5,50\}$ symbols. For comparison: BER with no spectral estimation, perfect spectral estimation, and no interference (thick dash-dotted lines). 


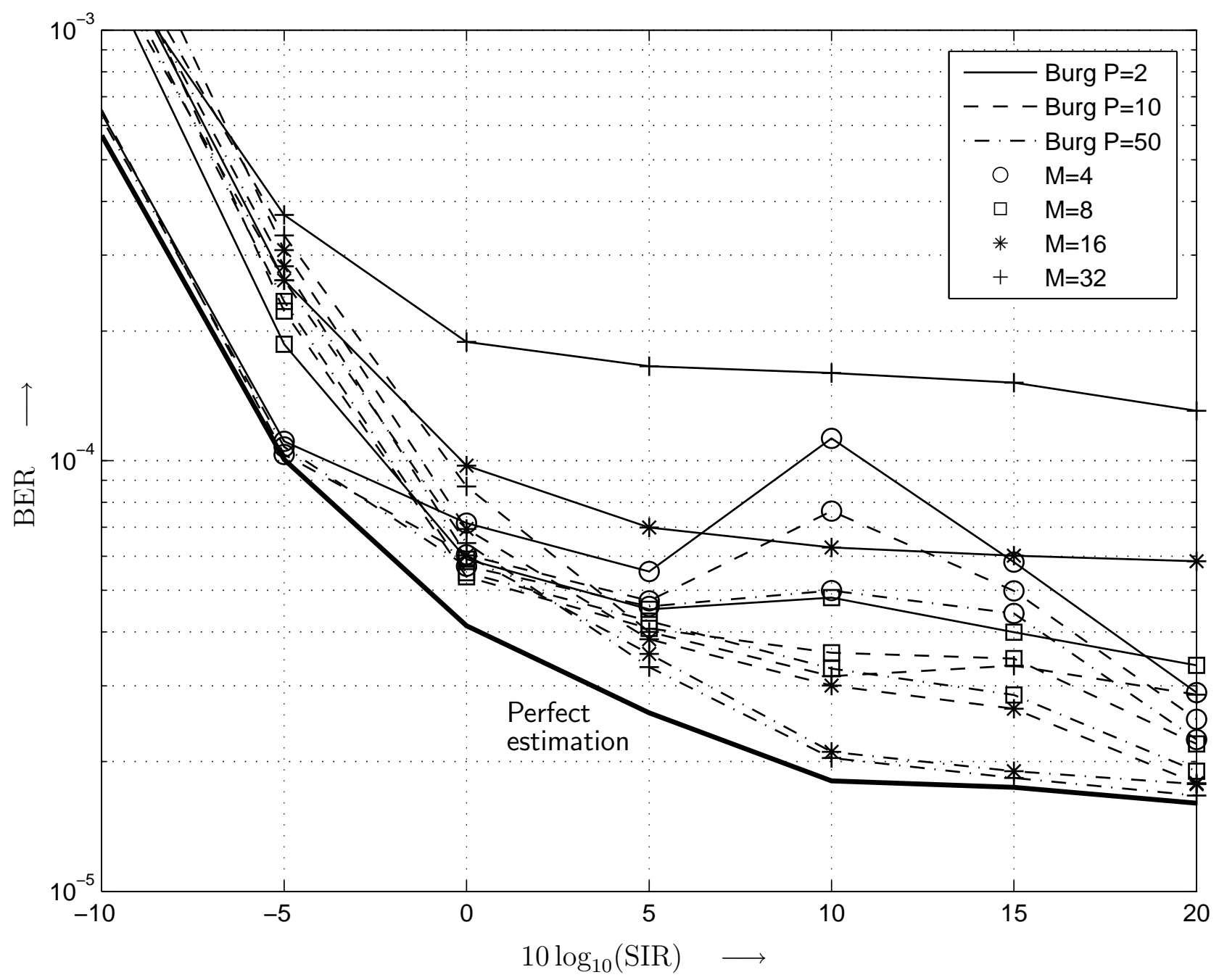

Figure 8: BER versus $10 \log _{10}(\mathrm{SIR})$ for $10 \log _{10}(\mathrm{SNR})=4.1$. MB-OFDM coded transmission with rate $1 / 2$. QPSK WiMAX-OFDM bandwidth $1.75 \mathrm{MHz}$, carrier frequency $f_{n}=3500 \mathrm{MHz}$. Nonfading WiMAX and MB-OFDM channels. Burg spectral estimation technique, AR model orders $M \in\{4,8,16,32\}$ and $P \in\{2,5,50\}$ symbols. For comparison: perfect spectral estimation (thick solid line). 


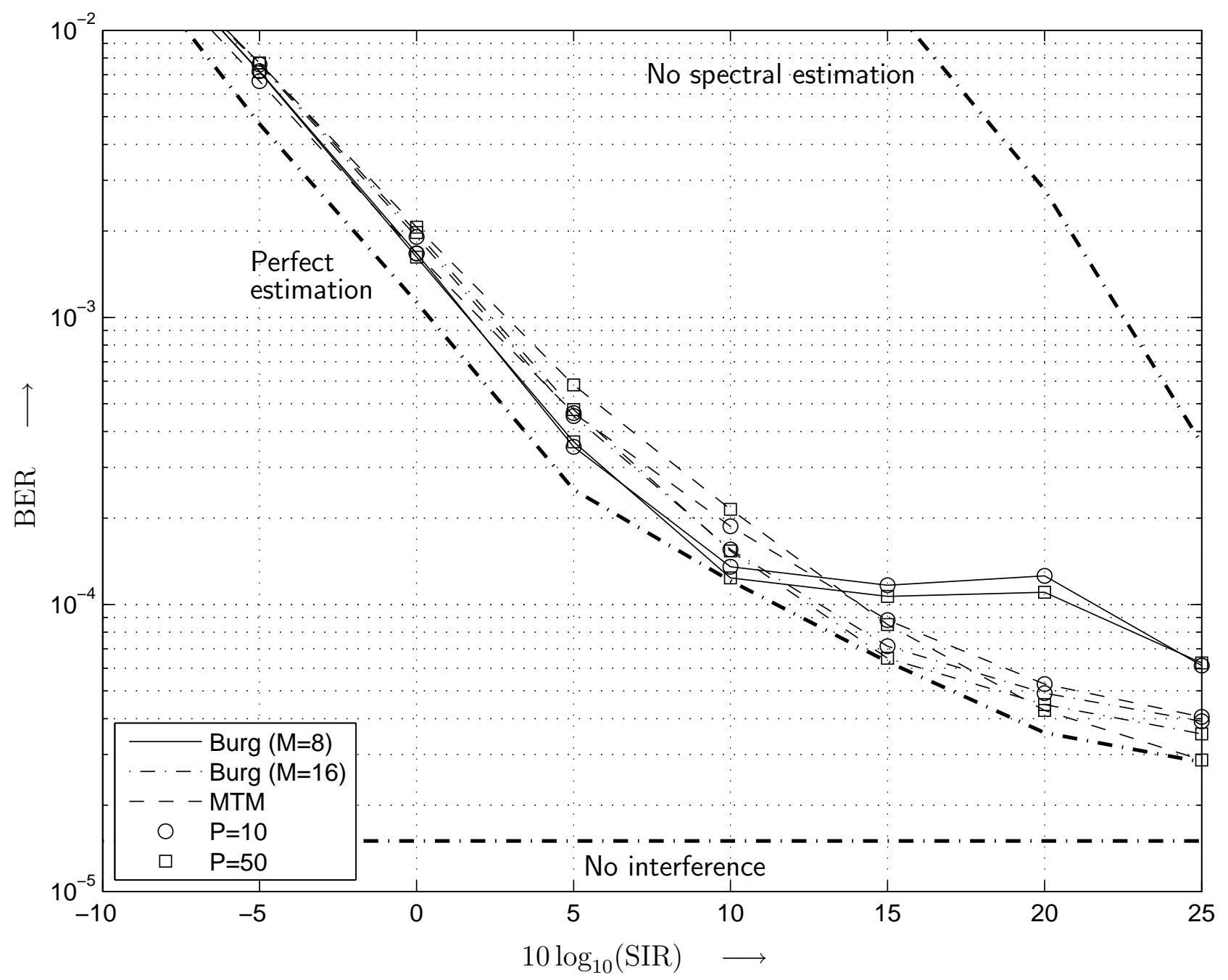

Figure 9: BER versus $10 \log _{10}(\mathrm{SIR})$ for $10 \log _{10}(\mathrm{SNR})=18$. MB-OFDM coded transmission with rate $3 / 4$. Two QPSK WiMAX-OFDM interferers with bandwidth $7 \mathrm{MHz}$, carrier frequencies $f_{n}=\{3475,3500\} \mathrm{MHz}$. UWB CM1 channel, non-fading WiMAX channel. Burg with $M \in\{8,16\}$ (solid, dash-dotted lines) and MTM (dashed lines) spectral estimation techniques, for $P \in\{10,50\}$ symbols. For comparison: BER with no spectral estimation, perfect spectral estimation, and no interference (thick dash-dotted lines). 